\title{
Efficient approximate analytical methods for nonlinear fuzzy boundary value problem
}

\author{
Ali Fareed Jameel ${ }^{1}$, Hafed H. Saleh ${ }^{2}$, Amirah Azmi ${ }^{2}$, Abedel-Karrem Alomari ${ }^{3}$, Nidal Ratib Anakira ${ }^{4}$, \\ Noraziah Haji Man ${ }^{1}$ \\ ${ }^{1}$ School of Quantitative Sciences, College of Arts and Sciences, Universiti Utara Malaysia, Kedah, Malaysia \\ ${ }^{2}$ School of Mathematical Sciences, Universiti Sains Malaysia, Gelugor, Penang, Malaysia \\ ${ }^{3}$ Department of Mathematics, Faculty of Science, Yarmouk University, Irbid, Jordan \\ ${ }^{4}$ Department of Mathematics, Faculty of Science and Technology, Irbid National University, Irbid, Jordan
}

\begin{abstract}
Article Info
Article history:

Received Feb 1, 2021

Revised Sep 15, 2021

Accepted Oct 10, 2021

Keywords:

Approximate analytical

methods

Fuzzy differential equations

Homotopy perturbation method

Two-point BVP

Variational iteration method

ABSTRACT

This paper aims to solve the nonlinear two-point fuzzy boundary value problem (TPFBVP) using approximate analytical methods. Most fuzzy boundary value problems cannot be solved exactly or analytically. Even if the analytical solutions exist, they may be challenging to evaluate. Therefore, approximate analytical methods may be necessary to consider the solution. Hence, there is a need to formulate new, efficient, more accurate techniques. This is the focus of this study: two approximate analytical methods-homotopy perturbation method (HPM) and the variational iteration method (VIM) is proposed. Fuzzy set theory properties are presented to formulate these methods from crisp domain to fuzzy domain to find approximate solutions of nonlinear TPFBVP. The presented algorithms can express the solution as a convergent series form. A numerical comparison of the mean errors is made between the HPM and VIM. The results show that these methods are reliable and robust. However, the comparison reveals that VIM convergence is quicker and offers a swifter approach over HPM. Hence, VIM is considered a more efficient approach for nonlinear TPFBVPs.
\end{abstract}

This is an open access article under the CC BY-SA license.

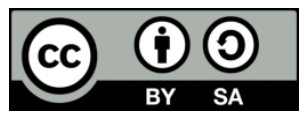

\section{Corresponding Author:}

Amirah Azmi

School of Mathematical Sciences, Universiti Sains Malaysia

Gelugor 11800 Penang, Malaysia

Email: amirahazmi@usm.my

\section{INTRODUCTION}

Real-life applications investigate the meaning of fuzzy as a generalization of crisp common sense because it is a solid instrument for modeling the vagueness, and in specific, to treat uncertainty with a mathematical model [1]. In many real well-determined dynamic issues, a system of ordinary or partial differential equations may represent the mathematical model. On the contrary, fuzzy differential equations (FDEs) are a valuable tool to model a dynamic system that is ambiguous in its existence and comportments. Since the FDEs have been used widely to model scientific and engineering problems, they have become a popular topic among researchers [2]. There are many practical problems with the solution of FDEs that satisfy initial [3] or boundary [4] values conditions. The main reason why finding the approximate solutions to the fuzzy problems becomes necessary is that most of the problems are too complicated to be solved exactly, or there are no analytical solutions at all [4]. Hence, FDEs will be suitable mathematical models for dynamic systems where complexity and ambiguity occur. For this reason, we may find FDEs exist in several fields of mathematics and science, including population models [5]-[7] and mathematical biology and 
physics [8]-[10]. As an alternative to the analytical solutions to such problems, approximate solutions such as homotopy perturbation method (HPM) and variational iteration method (VIM), and Adomian decomposition method (ADM) are listed as some of the approximate analytical methods [11].

In the last decade, some researchers have started to explore the numerical solutions for two-point fuzzy boundary value problem (TPFBVP) [12], [13]. Semi-analytical approaches have been used over recent years to overcome linear TPFBVP by various methods [14]-[16]. He implemented the HPM in 1999, and the method was applied to a wide range of mathematical and physical problems [17], [18]. This method provides the solution into components of a short convergence series, which are elegantly determined. HPM is now known as a standard tool for overcoming all kinds of linear and nonlinear equations, such as differential or integral equations. Another significant advantage is that the measurement size can be reduced while increasing the exactness of approximate solutions, so it is regarded as a robust process. Along with the HPM, VIM was introduced and also proposed by He [19]. This approach differs from specific classical techniques utilizing which nonlinear equations are quickly and accurately resolved. VIM has been used in many physics and engineering sectors recently [20], [21]. This approach is helpful for directly solving linear and nonlinear problems with $n^{\text {th }}$ order boundary value problems (BVPs) without reducing them to a BVP system. It has been reported by many authors, such as [22], that VIM is more robust than other analytical approaches, like ADM and HPM. Compared to HPM and ADM, where computer algorithms are commonly used for nonlinear terms, VIM is used explicitly without any nonlinear terms requirement or restrictive assumptions [23]. Without restrictive assumptions, the VIM solves differential equations that can change the structure of solutions. In VIM, the calculation is simple and straightforward [24]. The VIM overcomes the difficulty of measuring Adomian polynomials [23], a significant advantage over ADM.

According to [24], one significant disadvantage of VIM is that the terms obtained are longer than those obtained by decomposition and perturbation methods. For this reason, we are seeking and investigating the proposed approximate analytical solutions for nonlinear FDEs by HPM and VIM for comparison purposes. This study will develop an innovative approach to modifying the nonlinear TPFBVP based on the framework of fuzzy problems. This modification is tested on two existing FDEs and compared with the exact solution and the numerical solution. A comparative study will be given to show the capabilities of the proposed methods. According to the results, the modified schemes were found to be feasible and more accurate.

\section{PRELIMINARIES}

In this section, we provide some fundamental concepts and definitions that are necessary for this work. This includes some propositions, properties, and explanations of fuzzy sets and numbers and FDEs that will be used later in this work.

Definition 2.1 [25]: the relation:

$$
\mu(x ; \alpha, \beta, \gamma)=\left\{\begin{aligned}
0, & \text { if } x<\alpha \\
\frac{x-\alpha}{\beta-\alpha}, & \text { if } \alpha \leq x \leq \beta \\
\frac{\gamma-x}{\gamma-\beta}, & \text { if } \beta \leq x \leq y \\
0, & \text { if } x>\gamma
\end{aligned}\right.
$$

is a form of the membership function for a trapezoidal fuzzy number $\mu=(x ; \alpha, \beta, \gamma)$, which is presented as shown in Figure 1.

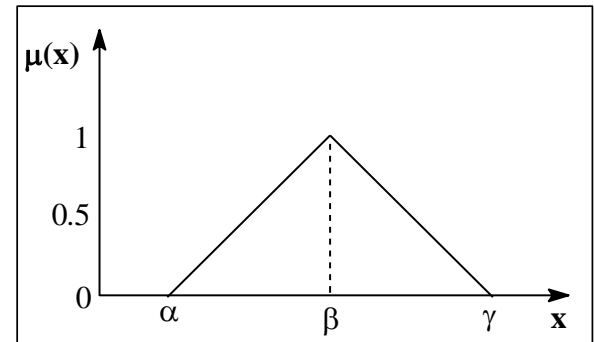

Figure 1. Triangular fuzzy number 
And its $r$-level is: $[\mu]_{r}=[\alpha+r(\beta-\alpha), \gamma-r(\gamma-\beta)], r \in[0,1]$.

Definition 2.2 [25]: The relation

$$
\mu(x ; \alpha, \beta, \gamma, \delta)= \begin{cases}0, & \text { if } x<\alpha \\ \frac{x-\alpha}{\beta-\alpha}, & \text { if } \alpha \leq x \leq \beta \\ 1, & \text { if } \beta \leq x \leq y \\ \frac{\delta-x}{\delta-\gamma}, & \text { if } y \leq x \leq \delta \\ 0, & \text { if } x>\delta\end{cases}
$$

Is a form of the membership function for a trapezoidal fuzzy number $\mu=(x ; \alpha, \beta, \gamma, \delta)$, which is presented as in Figure 2.

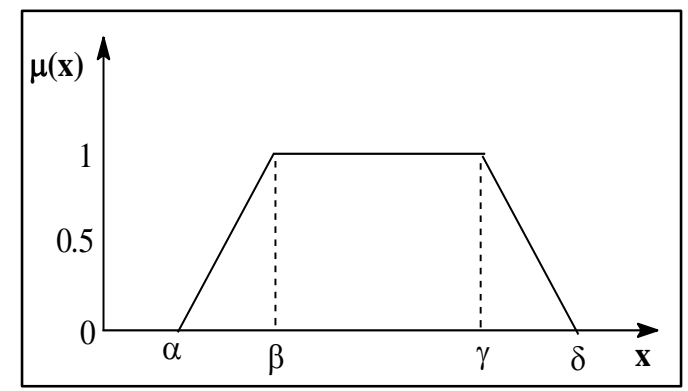

Figure 2. Trapezoidal fuzzy number

This can be used to describe an r-level set of the trapezoidal fugitive number as;

$$
[\tilde{\mu}]_{r}=[(\beta-\alpha) r+\alpha, \delta-(\delta-\gamma) r]
$$

This paper describes the class of all fuzzy subsets of $\mathbb{R}$ is being marked by $\tilde{E}$ that satisfies the characteristics fuzzy number properties [26].

Definition 2.3 [27]: Let $\tilde{f}: \mathbb{R} \rightarrow \tilde{E}, \tilde{f}(x)$ is called fuzzy function if $\tilde{E}$ is a set of fuzzy numbers.

Definition 2.4 [28]: The r-level set defined as $[\tilde{f}(x)]_{r}=[\underline{f}(x ; r), \bar{f}(x ; r)], x \in K, r \in[0,1]$ for a fuzzy function $f: T \rightarrow \tilde{E}$ where $T \subseteq \tilde{E}$. A fuzzy number is more effective than the r-level sets as representational types of fuzzy sets. Fuzzy sets can be described based on the resolution identity theorem by the families in their r-level sets.

Definition 2.5 [29]: If $f: X \rightarrow Y$ is function induces another function $\tilde{f}: F(X) \rightarrow F(Y)$ For each interval, $U$ in $X$ is defined by:

$$
\tilde{f}(U)(y)=\left\{\begin{array}{l}
\operatorname{Sup}_{x \in f^{-1}(y)} U(x), \text { if } y \in \operatorname{range}(f) \\
0, \text { if } y \notin \operatorname{range}(f)
\end{array}\right.
$$

This is recognized as the theory of Zadeh extension principle.

Definition 2.6 [30]: Let $\tilde{z}=\tilde{x} \ominus \tilde{y}$ be the H-difference of the fuzzy numbers $\tilde{x}$ and $\tilde{y}$ if the fuzzy number $\tilde{z}$ exist with the property $\tilde{x}=\tilde{y}+\tilde{z}$.

Definition 2.7 [13]: If $\tilde{f}: I \rightarrow \widetilde{E}$ and $t_{0} \in I$, where $I \in[a, b] . \widetilde{f}^{\prime}$ is said to be Hukuhara differentiable at $t_{0}$, if there exists an element $\left[\tilde{f}^{\prime}\right]_{r} \in \tilde{E}$ to be small enough for all $h>0$ (near to 0 ), exists $\tilde{f}\left(t_{0}+h ; r\right) \Theta$ $\tilde{f}\left(t_{0} ; r\right), \tilde{f}\left(t_{0} ; r\right) \ominus \tilde{f}\left(t_{0}-h ; r\right)$ and limits in metric $(\tilde{E}, D)$ are taken and exist in such a way that

$$
\widetilde{f}^{\prime}\left(t_{0}\right)=\lim _{h \rightarrow 0+} \frac{\tilde{f}\left(t_{0}+h ; r\right) \Theta \tilde{f}\left(t_{0} ; r\right)}{h}=\lim _{h \rightarrow 0+} \frac{\tilde{f}\left(t_{0} ; r\right) \Theta \tilde{f}\left(t_{0}-h ; r\right)}{h} .
$$

For more details, see [30]. 
Definition 2.8 [31]: Settle $\tilde{f}: I \rightarrow \tilde{E}$ and $t_{0} \in I$, for $\in[a, b] . \tilde{f}^{(n)}$ is said to be Hukuhara differentiable $x \in \tilde{E}$, if there exists an element $\left[\tilde{f}^{(n)}\right]_{r} \in \tilde{E}$ to be small enough for all $h>0$ (near to 0), exists $\widetilde{f}^{(n-1)}\left(t_{0}+h ; r\right) \ominus \tilde{f}^{(n-1)}\left(t_{0} ; r\right), \tilde{f}^{(n-1)}\left(t_{0} ; r\right) \ominus \tilde{f}^{(n-1)}\left(t_{0}-h ; r\right)$ and limits in metric $(\tilde{E}, D)$ are taken and exist in such a way that

$$
\tilde{f}^{(n)}\left(t_{0}\right)=\lim _{h \rightarrow 0+} \frac{\tilde{f}^{(n-1)}\left(t_{0}+h ; r\right) \Theta \tilde{f}^{(n-1)}\left(t_{0} ; r\right)}{h}=\lim _{h \rightarrow 0+} \frac{\tilde{f}^{(n-1)}\left(t_{0} ; r\right) \Theta \tilde{f}^{(n-1)}\left(t_{0}-h ; r\right)}{h}
$$

There is a second order of the derivatives of Hukuhara for $n=2$ and equivalent to $\tilde{f}^{(n)}$.

Theorem 2.1 [31]: Let $\tilde{f}:[t+\alpha, b] \rightarrow \tilde{E}$ be Hukuhara differentiable and denote

$$
\left[\widetilde{f}^{\prime}(t)\right]_{r}=\left[\underline{f}^{\prime}(t), \bar{f}^{\prime}(t)\right]_{r}=\left[\underline{f}^{\prime}(t ; r), \bar{f}^{\prime}(t ; r)\right]
$$

Then we can define the differentiable boundary functions $f^{\prime(t ; r)}$ and $\bar{f}^{\prime}(t ; r)$ can be written in the $\mathrm{n}^{\text {th }}$ order of $\mathrm{n}^{\text {th }}$ fuzzy derivatives.

$$
\left[\tilde{f}^{(n)}(t)\right]_{r}=\left[\left(\underline{f}^{(n)}(t ; r)\right),\left(\bar{f}^{(n)}(t ; r)\right)\right], \forall r \in[0,1]
$$

\section{DESCRIPTION OF THE FUZZY HPM}

The overall HPM structure for solving crisp nonlinear TFBVP is mentioned in [17], [18]. Consider the defuzzification of the following general $\mathrm{n}^{\text {th }}$ order TPFBVP [15].

$$
\begin{aligned}
& \tilde{y}^{(n)}(t)=f\left(t, \tilde{y}(t), \tilde{y}^{\prime}(t), \tilde{y}^{\prime \prime}(t), \ldots \tilde{y}^{(n-1)}(t)\right)+\tilde{g}(t), \quad t \in\left[t_{0}, T\right] \\
& \left\{\begin{array}{l}
\tilde{y}\left(t_{0}\right)=\tilde{\alpha}^{(0)}, \tilde{y}^{\prime}\left(t_{0}\right)=\tilde{\alpha}^{(1)}, \ldots . \tilde{y}^{(k)}\left(t_{0}\right)=\tilde{\alpha}^{(k)} \\
\tilde{y}(T)=\tilde{\beta}^{(0)}, \tilde{y}^{\prime}(T)=\tilde{\beta}^{(1)}, \ldots ., \tilde{y}^{(n-k-2)}(T)=\tilde{\beta}^{(n-k-2)},
\end{array}\right.
\end{aligned}
$$

To solve (1) by using HPM, we need to fuzzify HPM and then defuzzify it back to (1) as in [15]. According to [32], the HPM and for all $r \in[0,1]$. HPM must ensure the convergence of the HPM solution series function through the correct choice of initial guess and the auxiliary linear operator [33]. From [15], HPM form for solving (1) is given by solving the lower bound as follows:

$$
\begin{aligned}
& p^{0}:\left\{\begin{array}{c}
\underline{L}_{n}\left[\underline{y}_{0}(t ; r)-\underline{y}_{0}\left(t ; r ; \sum_{s=1}^{n} \underline{c}_{s}(r)\right)\right]=0, \\
\underline{y}\left(t_{0} ; r\right)=\left[\underline{\alpha}_{0}\right]_{r}, \underline{y}^{\prime}\left(t_{0} ; r\right)=\left[\underline{\alpha}_{1}\right]_{r}, \ldots, \underline{y}^{(n-1)}\left(t_{0} ; r\right)=\left[\underline{\alpha}_{n-1}\right]_{r} \\
\underline{y}(T ; r)=\left[\underline{\beta}_{0}\right]_{r}, \underline{y}^{\prime}(T ; r)=\left[\underline{\beta}_{1}\right]_{r}, \ldots, \underline{y}^{(n-k-2)}(T ; r)=\left[\underline{\beta}_{n-k-2}\right]_{r} .
\end{array}\right. \\
& p^{1}:\left\{\begin{array}{c}
\underline{L}_{n}\left[\underline{y}_{1}(t ; r)+\underline{y}_{0}\left(t ; r ; \sum_{s=1}^{n} \underline{c}_{s}(r)\right)\right]-F\left(t, \tilde{V}_{0}\left(t ; r, \sum_{i=1}^{n} \underline{c}_{s}(r)\right)\right) \\
-\underline{g}(t ; r)=0, \\
\underline{y_{1}}\left(t_{0} ; r\right)=0_{r}, \underline{y}_{1}{ }^{\prime}\left(t_{0} ; r\right)=0, \ldots, \underline{y}_{1}{ }^{(n-1)}\left(t_{0} ; r\right)=0, \\
\underline{y}_{1}(T ; r)=0_{r}, \underline{y}_{1}{ }^{\prime}(T ; r)=0, \ldots, \underline{y}_{1}{ }^{(n-k-2)}(T ; r)=0 .
\end{array}\right. \\
& p^{2}:\left\{\begin{array}{c}
\underline{L}_{n} \underline{y}_{2}(t ; r)-F\left(t, \tilde{V}_{1}\left(t ; r, \sum_{i=1}^{n} \underline{c}_{s}(r)\right)\right)=0, \\
\underline{y}_{2}\left(t_{0} ; r\right)=0_{r}, \underline{y}_{2}^{\prime}\left(t_{0} ; r\right)=0, \ldots, \underline{y}_{2}{ }^{(n-1)}\left(t_{0} ; r\right)=0, \\
\underline{y}_{2}(T ; r)=0_{r}, \underline{y}_{2}{ }^{\prime}(T ; r)=0, \ldots, \underline{y}_{2}{ }^{(n-k-2)}(T ; r)=0 .
\end{array}\right. \\
& \vdots \\
& p^{n+1}:\left\{\begin{array}{c}
\underline{L}_{n} \underline{y}_{n+1}(t ; r)-F\left(t, \tilde{V}_{n}\left(t ; r, \sum_{i=1}^{n} \underline{c}_{s}(r)\right)\right)=0, \\
\underline{y}_{n+1}\left(t_{0} ; r\right)=0_{r}, \underline{y}_{n+1}{ }^{\prime}\left(t_{0} ; r\right)=0, \ldots, \underline{y}_{n+1}{ }^{(n-1)}\left(t_{0} ; r\right)=0, \\
\underline{y}_{n+1}(T ; r)=0_{r}, \underline{y}_{n+1}{ }^{\prime}(T ; r)=0, \ldots, \underline{y}_{n+1}{ }^{(n-k-2)}(T ; r)=0 .
\end{array}\right.
\end{aligned}
$$


Similarly for the upper bound

$$
\begin{aligned}
& p^{0}:\left\{\begin{array}{c}
\bar{L}_{n}\left[\bar{y}_{0}(t ; r)-\bar{y}_{0}\left(t ; r ; \sum_{s=1}^{n} \bar{c}_{s}(r)\right)\right]=0, \\
\bar{y}\left(t_{0} ; r\right)=\left[\bar{\alpha}_{0}\right]_{r}, \bar{y}^{\prime}\left(t_{0} ; r\right)=\left[\bar{\alpha}_{1}\right]_{r}, \ldots, \bar{y}^{(n-1)}\left(t_{0} ; r\right)=\left[\bar{\alpha}_{n-1}\right]_{r}, \\
\bar{y}(T ; r)=\left[\bar{\beta}_{0}\right]_{r}, \bar{y}^{\prime}(T ; r)=\left[\bar{\beta}_{1}\right]_{r}, \ldots, \bar{y}^{(n-k-2)}(T ; r)=\left[\bar{\beta}_{n-k-2}\right]_{r} .
\end{array}\right. \\
& p^{1}:\left\{\begin{array}{c}
\bar{L}_{n}\left[\bar{y}_{1}(t ; r)+\bar{y}_{0}\left(t ; r ; \sum_{s=1}^{n} \bar{c}_{s}(r)\right)\right]-G\left(t, \tilde{V}_{0}\left(t ; r, \sum_{i=1}^{n} \bar{c}_{s}(r)\right)\right), \\
-\bar{g}(t ; r)=0, \\
\bar{y}_{1}\left(t_{0} ; r\right)=0, \bar{y}_{1}^{\prime}\left(t_{0} ; r\right)=0, \ldots, \bar{y}_{1}^{(n-1)}\left(t_{0} ; r\right)=0, \\
\bar{y}_{1}(T ; r)=0, \bar{y}_{1}^{\prime}(T ; r)=0, \ldots, \bar{y}_{1}^{(n-k-2)}(T ; r)=0 .
\end{array}\right. \\
& p^{2}:\left\{\begin{array}{c}
\bar{L}_{n} \bar{y}_{2}(t ; r)-G\left(t, \tilde{V}_{n}\left(t ; r, \sum_{i=1}^{n} \bar{c}_{s}(r)\right)\right)=0, \\
\bar{y}_{2}\left(t_{0} ; r\right)=0, \bar{y}_{2}^{\prime}\left(t_{0} ; r\right)=0, \ldots, \bar{y}_{2}{ }^{(n-1)}\left(t_{0} ; r\right)=0, \\
\bar{y}_{2}(T ; r)=0, \bar{y}_{2}{ }^{\prime}(T ; r)=0, \ldots, \bar{y}_{2}{ }^{(n-k-2)}(T ; r)=0 .
\end{array}\right. \\
& p^{n+1}:\left\{\begin{array}{c}
\bar{L}_{n} \bar{y}_{n+1}(t ; r)-G\left(t, \tilde{V}_{n}\left(t ; r, \sum_{i=1}^{n} \bar{c}_{s}(r)\right)\right)=0, \\
\bar{y}_{n+1}\left(t_{0} ; r\right)=0, \bar{y}_{n+1}{ }^{\prime}\left(t_{0} ; r\right)=0, \ldots, \bar{y}_{n+1}^{(n-1)}\left(t_{0} ; r\right)=0, \\
\bar{y}_{n+1}(T ; r)=0, \bar{y}_{n+1}{ }^{\prime}(T ; r)=0, \ldots, \bar{y}_{n+1}^{(n-k-2)}(T ; r)=0,
\end{array}\right.
\end{aligned}
$$

where $\tilde{V}(t)=\tilde{y}(t), \tilde{y}^{\prime}(t), \tilde{y}^{\prime \prime}(t), \ldots \tilde{y}^{(n-1)}(t)$ and the initials guessing $\tilde{y}\left(t_{0} ; r\right)$ is given in [16] for all $r \in$ $[0,1]$ and then the approximate solution is given by setting $p=1$ as in (3):

$$
\tilde{y}\left(t ; r ; \sum_{s=1}^{n} \tilde{c}_{s}(r)\right)=S_{m}\left(t ; r ; \sum_{s=1}^{n} \tilde{c}_{s}(r)\right)=\sum_{i=0}^{m-1} \tilde{V}_{i}\left(t ; r ; \sum_{s=1}^{n} \tilde{c}_{s}(r)\right)
$$

Therefore, the exact solution of (1) can now be obtained by setting $\mathrm{p}=1$ :

$$
\begin{aligned}
& \tilde{Y}(t ; r)=\lim _{p \rightarrow 1} \tilde{y}\left(t ; r ; \sum_{s=1}^{n} \tilde{c}_{s}(r)\right)=\lim _{p \rightarrow 1}\left\{\sum_{i=0}^{\infty} p^{i} \tilde{V}_{i}\left(t ; r ; \sum_{s=1}^{n} \tilde{c}_{s}(r)\right)\right\} \\
& =\sum_{i=0}^{\infty} \tilde{V}_{i}\left(t ; r ; \sum_{s=1}^{n} \tilde{c}_{s}(r)\right)
\end{aligned}
$$

\section{DESCRIPTION OF THE FUZZY VIM}

VIM general structure for resolving problems TFPBVP is stated in [19]. In order for the (1) to be solved using VIM, we must fuzzify and defuzzify the VIM as defined in (2) [34]. According to VIM in [22] and for all $r \in[0,1]$ we rewrite (1) in the following correction functional forms:

$$
\begin{aligned}
& \underline{y_{i+1}}\left(t ; r ; \sum_{s=1}^{n} \underline{c}_{s}(r)\right)=\underline{y}_{i}\left(t ; r ; \sum_{s=1}^{n} \underline{c}_{S}(r)\right)+ \\
& \int_{0}^{t} \lambda(t ; \eta)\left\{\underline{y}_{i}^{(n)}(\eta ; r)+F(\eta, \tilde{\tilde{V}}(\eta ; r))+\underline{g}(\eta ; r)\right\} d \eta, \\
& \bar{y}_{i+1}\left(t ; r ; \sum_{s=1}^{n} \bar{c}_{s}(r)\right) \\
& =\bar{y}_{i}\left(t ; r ; \sum_{s=1}^{n} \bar{c}_{s}(r)\right)+\int_{0}^{t} \lambda(t ; \eta)\left\{\bar{y}_{i}^{(n)}(\eta ; r)+G(\eta, \tilde{\tilde{V}}(\eta ; r))+\bar{g}(\eta ; r)\right\} d \eta,
\end{aligned}
$$

where $i=1,2, \ldots, r \in[0,1]$. The Lagrange multiplier is $\lambda(t ; \eta)$ which can be optimally defined via variational theory [33]. Now we let

$$
\begin{aligned}
& F_{*} \tilde{\widetilde{y}}_{l}=F(\eta, \tilde{\tilde{V}}(\eta ; r))+\underline{g}(\eta ; r), \\
& G_{*} \tilde{\widetilde{y_{l}}}=G(\eta, \tilde{\tilde{V}}(\eta ; r))+\bar{g}(\eta ; r),
\end{aligned}
$$


where $F_{*}$ and $G_{*}$ are nonlinear operators including the nonlinear terms $F$ and $G$ and the inhomogeneous term $\tilde{g}(\eta ; r)$. In the following forms we will rewrite (5) and (6) as:

$$
\begin{aligned}
& \underline{y}_{i+1}\left(t ; r ; \sum_{s=1}^{n} \underline{c}_{s}(r)\right)=\underline{y}_{i}\left(t ; r ; \sum_{s=1}^{n} \underline{c}_{s}(r)\right)+\int_{0}^{t} \lambda(t ; \eta)\left\{\underline{y}_{i}^{(n)}(\eta ; r)+F_{*} \tilde{\widetilde{y}}_{l}\right\} d \eta, \\
& \bar{y}_{i+1}\left(t ; r ; \sum_{s=1}^{n} \bar{c}_{s}(r)\right)=\bar{y}_{i}\left(t ; r ; \sum_{s=1}^{n} \bar{c}_{s}(r)\right)+\int_{0}^{t} \lambda(t ; \eta)\left\{\bar{y}_{i}^{(n)}(\eta ; r)+G_{*} \tilde{\widetilde{y}}_{l}\right\} d \eta,
\end{aligned}
$$

where restricted variation is $\tilde{\tilde{y}_{l}}$, i.e $\delta \tilde{\tilde{y}_{l}}=0$ [21]. The general multiplier $\lambda(t ; \eta)$ applied to (1) according to [23] can be described in the following:

$$
\begin{aligned}
& \delta \underline{y}_{i+1}\left(t ; r ; \sum_{s=1}^{n-1} \underline{c}_{s}(r)\right)=\delta \underline{y}_{i}\left(t ; r ; \sum_{s=1}^{n-1} \underline{c}_{s}(r)\right)+\delta \int_{0}^{t} \lambda(t ; \eta)\left\{\underline{y}_{i}^{(n)}(\eta ; r)+F_{*} \widetilde{\widetilde{y}}_{l}\right\} d \eta \\
& =\delta \underline{y}_{i+1}\left(t ; r ; \sum_{s=1}^{n-1} \underline{c}_{s}(r)\right)=\delta \underline{y}_{i}\left(t ; r ; \sum_{s=1}^{n-1} \underline{c}_{s}(r)\right)+\delta \int_{0}^{t} \lambda(t ; \eta)\left\{\underline{y}_{i}^{(n)}(\eta ; r)\right\} d \eta .
\end{aligned}
$$

According to [23], we obtain the followings by integrating by part:

$$
\begin{aligned}
& \underline{y}_{i+1}\left(t ; r ; \sum_{s=1}^{n-1} \underline{c}_{s}(r)\right)=\left[1-\lambda(t)^{(n-1)}\right] \delta \underline{y}_{i}\left(t ; r ; \sum_{s=1}^{n-1} \underline{c}_{s}(r)\right)+\sum_{k=}^{n-2} \delta \underline{y}_{i}^{(n-1-k)}(t ; r)+ \\
& \int_{0}^{t} \lambda(t ; \eta)^{(n)} \delta \underline{y_{i}}\left(t ; r ; \sum_{s=1}^{n-1} \underline{c}_{s}(r)\right) d \eta .
\end{aligned}
$$

The following stationary conditions can therefore be reached [35].

$$
\left\{\begin{array}{l}
\lambda(\eta)^{(n)}=0, \\
1-\lambda(t)^{(n-1)}=0, \\
\lambda(t)^{(k)}=0, k=0,1,2, \ldots, n-2 .
\end{array}\right.
$$

Under these terms and the order of the (1) the general Lagrangian multiplier can be calculated as [35]:

$$
\left.\lambda(t ; \eta)=\frac{(-1)^{n}(\eta-t)^{n-1}}{(n-1) !}\right)
$$

Therefore, all the above parameters $\lambda(t ; \eta)$ and $\tilde{y}_{0}\left(t ; r ; \sum_{s=1}^{n} \tilde{c}_{s}(r)\right)$ will be easily obtained in the series of approximations of VIM. The exact solution can therefore be obtained:

$$
\tilde{y}(t ; r)=\lim _{i \rightarrow 1} \tilde{y}_{i}\left(t ; r ; \sum_{s=1}^{n} \tilde{c}_{s}(r)\right) \text {. }
$$

To determine the $\tilde{c}_{S}(r)$, we use the same HPM technique as in [15] by substituting these constants with the initial estimates in series solution function and then using the boundary conditions of (1) to determine the values of these constants for each fuzzy $r$-level set.

\section{NUMERICAL EXAMPLES}

The approximate solution by HPM and VIM in the next examples are obtained by formulating the given equations as presented in sections 3 and 4 . The formula is then solved and analyzed by using Mathematica 11:

Example 5.1: Let us consider the following nonlinear second order TPFBVP:

$$
\tilde{y}^{\prime \prime}(t)+\tilde{y}^{2}(t)=t^{4}+2, \bar{y}(0)=\tilde{a}, \tilde{y}(1)=\tilde{b}
$$

where $\tilde{a}$ and $\tilde{b}$ are triangular fuzzy numbers having r-level sets $[0.1 r-0.1,0.1-0.1 r]$ and $[0.9+0.1 r, 1.1-0.1 r]$ for all $r \in[0,1]$. The linear operator in compliance with section 2 is $\tilde{L}_{2}=\frac{d^{(2)}}{d t^{2}}$ with the inverse operator $\tilde{L}_{2}{ }^{-1}$, and the initial guesses for all $r \in[0,1]$ are given by:

$$
\left\{\begin{array}{l}
y_{0}(t ; r)=\underline{c}_{1}(r)+\underline{c}_{2}(r) t, \\
\bar{y}_{0}(t ; r)=\bar{c}_{1}(r)+\bar{c}_{2}(r) t .
\end{array}\right.
$$




\subsection{HPM formulation} of (7) are

According to section 3 , the values of $\tilde{c}_{1}(r)=[0.1 r-0.1,0.1-0.1 r]$ and the homotopy functions

$$
\begin{aligned}
& \underline{\mathcal{H}}(t, p ; r)=(1-p) \underline{L}_{2}\left[\underline{y}(t ; r)-\underline{y}_{0}\left(t ; r ; \underline{c}_{2}(r)\right)\right]+p\left[\underline{L}_{2} \underline{y}(t ; r)+(\underline{y}(t ; r))^{2}-\left(t^{4}+2\right)\right]=0, \\
& \underline{\mathcal{H}}(t, p ; r)=(1-p) \underline{L}_{2}\left[\underline{y}(t ; r)-\underline{y}_{0}\left(t ; r ; \underline{c}_{2}(r)\right)\right]+p\left[\underline{L}_{2} \underline{y}(t ; r)+(\underline{y}(t ; r))^{2}-\left(t^{4}+2\right)\right]=0, \\
& \overline{\mathcal{H}}(t, p ; r)=(1-p) \bar{L}_{2}\left[\bar{y}(t ; r)-\bar{y}_{0}\left(t ; r ; \bar{c}_{2}(r)\right)\right]+p\left[L_{2} \bar{y}(t ; r)+(\bar{y}(t ; r))^{2}-\left(t^{4}+2\right)\right]=0 .
\end{aligned}
$$

The $n$ components are specified as the HPM in section 3 of $\tilde{y}_{k}(t ; r)$ for $k=1,2, \ldots, n$ and $r \in[0,1]$ calculated by evaluating the lower limit as follows:

$$
\begin{aligned}
& p^{0}:\left\{\underline{y}_{0}(t ; r)=0.1 r-0.1+\underline{c}_{2}(r) t,\right. \\
& p^{1}:\left\{\begin{array}{c}
\underline{y}_{1}(t ; r)=\underline{L}_{2}^{-1}\left[\left(-\underline{y}_{0}\left(t ; r ; \underline{c}_{2}(r)\right)\right)^{2}+t^{4}+2\right], \\
\underline{y}_{1}(0 ; r)=0, \underline{y}_{1}(1 ; r)=0 .
\end{array}\right. \\
& p^{2}:\left\{\begin{array}{c}
\underline{y}_{2}(t ; r)=-\underline{L}_{2}^{-1}\left[2 \underline{y}_{1}\left(t ; r ; \underline{c}_{2}(r)\right) \underline{y}_{0}\left(t ; r ; \underline{c}_{2}(r)\right)\right], \\
\underline{y}_{2}(0 ; r)=0, \underline{y}_{2}(1 ; r)=0 .
\end{array}\right. \\
& \vdots \\
& p^{k+1}:\left\{\begin{array}{c}
\underline{y}_{k+1}(t ; r)=-\underline{L}_{2}^{-1} \sum_{k=0}^{n-1} \underline{y}_{k}\left(t ; r ; \underline{c}_{2}(r)\right) \underline{y}_{n-1-k}\left(t ; r ; \underline{c}_{2}(r)\right), \\
\underline{y}_{k}(0 ; r)=0, \underline{y}_{k}(1 ; r)=0 .
\end{array}\right.
\end{aligned}
$$

For the upper limit, the values are obtained in the same way as follows:

$$
\begin{aligned}
& p^{0}:\left\{\bar{y}_{0}(t ; r)=0.1-0.1 r+\bar{c}_{2}(r) t,\right. \\
& p^{1}:\left\{\begin{array}{c}
\bar{y}_{1}(t ; r)=\bar{L}_{2}{ }^{-1}\left[-\left(\bar{y}_{0}\left(t ; r ; \bar{c}_{2}(r)\right)\right)^{2}+t^{4}+2\right], \\
\bar{y}_{1}(0 ; r)=0, \bar{y}_{1}(1 ; r)=0 .
\end{array}\right. \\
& p^{2}:\left\{\begin{array}{c}
\bar{y}_{2}(t ; r)=-\bar{L}_{2}^{-1}\left[2 \bar{y}_{1}\left(t ; r ; \bar{c}_{2}(r)\right) \bar{y}_{0}\left(t ; r ; \bar{c}_{2}(r)\right)\right], \\
\bar{y}_{2}(0 ; r)=0, \bar{y}_{2}(1 ; r)=0 .
\end{array}\right. \\
& \vdots \\
& p^{k+1}:\left\{\begin{array}{c}
\bar{y}_{k+1}(t ; r)=-\bar{L}_{2}{ }^{-1}\left[\sum_{k=0}^{n-1} \bar{y}_{k}\left(t ; r ; \bar{c}_{2}(r)\right) \bar{y}_{n-1-k}\left(t ; r ; \bar{c}_{2}(r)\right)\right], \\
\bar{y}_{k}(0 ; r)=0, \bar{y}_{k}(1 ; r)=0 .
\end{array}\right.
\end{aligned}
$$

Evaluating (8) and (9) to obtain fifth order HPM series solution in the following form such that

$$
\tilde{S}_{5}\left(t ; r ; \tilde{c}_{2}(r)\right)=\tilde{y}_{0}\left(t ; r, \tilde{c}_{2}(r)\right)+\sum_{i=1}^{5} \tilde{y}_{i}\left(t ; r ; \tilde{c}_{2}(r)\right)=\tilde{y}\left(t ; r ; \tilde{c}_{2}(r)\right)
$$

Now to obtain the values of $\tilde{c}_{2}(r)$ for all $r \in[0,1]$, we solve the nonlinear series solution of (7) from the boundary condition $[0.9+0.1 r, 1.1-0.1 r]$ then we substitute the values of $\tilde{c}_{2}(r)$ again in (10) to obtain fifth order HPM series solution. Since (7) is considered without exact analytical solution, to show the accuracy of fifth order HPM approximate series solution $\tilde{S}_{5}(t ; r)$ for all $r \in[0,1]$, the residual error must be specified:

$$
[\tilde{E}(t)]_{r}=\tilde{S}_{5}(t ; r)^{\prime \prime}(t)+\tilde{S}_{5}(t ; r)^{2}(t)-t^{4}+2
$$

Then the fifth order HPM series solution is presented in the Tables 1-2 and Figure 3. According to Tables 1 and 2 and Figure 3, we concluded that the fifth order HPM approximate solutions of (7) for all $t \in[0,1]$ and $r \in[0,1]$ fulfill the patterns of fuzzy numbers in the form of a triangular fuzzy number. 
Table 1. Approximate solution $y(t ; r)$ of fifth order HPM at $t=0.5$ and $r \in[0,1]$

\begin{tabular}{cccc}
\hline $\mathrm{r}$ & $\underline{c_{2}}(r)$ & $\underline{y}(0.5 ; r)$ & {$[\underline{E}(0.5)]_{r}$} \\
\hline 0 & -0.012233517979781578 & 0.14366068333554236 & $4.183283991154862 \times 10^{-7}$ \\
0.25 & -0.010209834659421881 & 0.16997259607001605 & $2.946362775008149 \times 10^{-7}$ \\
0.5 & -0.007505097713710612 & 0.19646322624295728 & $2.485028610110795 \times 10^{-7}$ \\
0.75 & -0.004110208069923338 & 0.22313542853095245 & $2.726826934945636 \times 10^{-7}$ \\
1 & -0.000016001865820840 & 0.24999204985486695 & $6.342108563664461 \times 10^{-7}$ \\
\hline
\end{tabular}

Table 2. Approximate solution $\bar{y}(t ; r)$ of fifth order HPM at $t=0.5$ and $r \in[0,1]$

\begin{tabular}{cccc}
\hline $\mathrm{r}$ & $\underline{c}_{2}(r)$ & $\underline{y}(0.5 ; r)$ & {$[\underline{E}(0.5)]_{r}$} \\
\hline 0 & 0.023538969063634813 & 0.35931908435924000 & 0.0000211620477442997 \\
0.25 & 0.016554919244886100 & 0.33169666727607905 & 0.0000108129304682291 \\
0.5 & 0.010307281584441760 & 0.30426986657956345 & 0.0000048897414700183 \\
0.75 & 0.004786740073139446 & 0.27703592414404127 & 0.0000018877716657511 \\
1 & -0.000016001865820840 & 0.24999204985486695 & $6.342108563664461 \times 10^{-7}$ \\
\hline
\end{tabular}

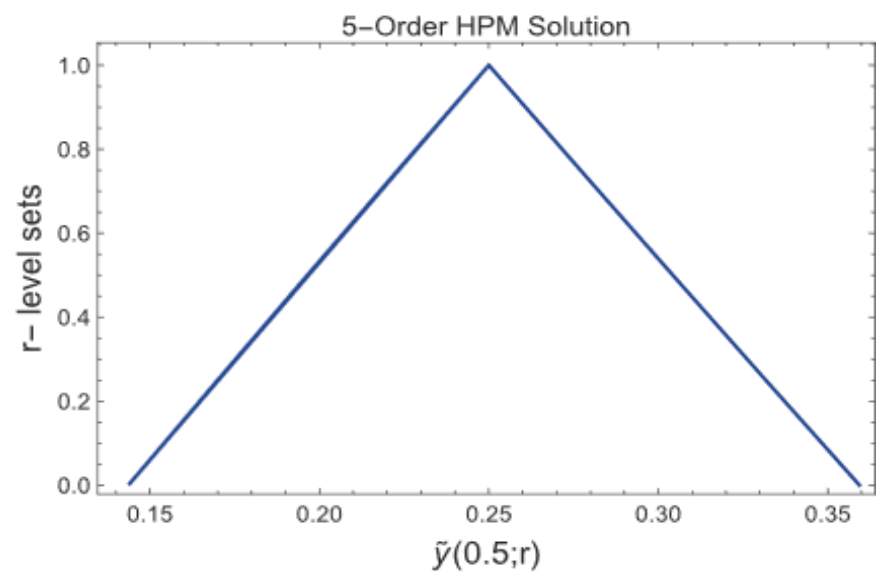

Figure 3. HPM approximate solution of (12) at $t=0.5$ and $r \in[0,1]$

\subsection{VIM formulation}

The variational formula of this problem is given in accordance with section 4 as (11):

$$
\left\{\begin{array}{c}
\underline{y}_{i+1}\left(t ; r ; \underline{c}_{2}(r)\right)=\underline{y_{i}}\left(t ; r ; \underline{c}_{2}(r)\right) \\
+\int_{0}^{t} \lambda(t ; \eta)\left\{\underline{y}_{i}^{\prime \prime}\left(\eta ; r ; \underline{c}_{2}(r)\right)+\underline{y}_{i}\left(\eta ; r ; \underline{c}_{2}(r)\right)^{2}-\eta^{4}-2\right\} d \eta \\
\bar{y}_{i+1}\left(t ; r ; \bar{c}_{2}(r)\right)=\bar{y}_{i}\left(t ; r ; \bar{c}_{2}(r)\right) \\
+\int_{0}^{t} \lambda(t ; \eta)\left\{\bar{y}_{i}^{\prime \prime}\left(\eta ; r ; \bar{c}_{2}(r)\right)+\bar{y}_{i}\left(\eta ; r ; \bar{c}_{2}(r)\right)^{2}-\eta^{4}-2\right\} d \eta .
\end{array}\right.
$$

The fourth-order VIM series solution is obtained in the form 12:

$$
\tilde{S}_{4}\left(t ; r ; \tilde{c}_{2}(r)\right)=\sum_{i=0}^{4} \tilde{y}_{i}\left(t ; r ; \tilde{c}_{2}(r)\right)=\tilde{y}(t ; r)
$$

The Lagrangian multiplier of (11) is described in section 4 such that $\lambda(t ; \eta)=\eta-t$. Now in order to obtain the values of $\tilde{c}_{2}(r)$ for all $r \in[0,1]$, we solve the nonlinear series solution of (7) from the boundary condition $[0.9+0.1 r, 1.1-0.1 r]$ then we substitute the values of $\tilde{c}_{2}(r)$ again in (12) to obtain fourth-order VIM series solution. For (1), the following residual error is described in order to demonstrate VIM accuracy in approximate fourth-order solution without an exact analytical solution:

$$
[\tilde{E}(t)]_{r}=\left|\tilde{S}_{4}^{\prime \prime}(t ; r)+\left[\tilde{S}_{4}(t ; r)\right]^{2}-t^{4}-2\right|
$$

In Table 3 and 4 and Figure 4, the fourth order VIM series solution is presented: 
Table 3. Approximate solution $y(t ; r)$ of fourth order VIM at $t=0.5$ and $r \in[0,1]$

\begin{tabular}{cccc}
\hline $\mathrm{r}$ & $\underline{c}_{2}(r)$ & $\underline{y}(0.5 ; r)$ & {$[\underline{E}(0.5)]_{r}$} \\
\hline 0 & -0.012227411850487582 & 0.14366373723600040 & $1.268180005453700 \times 10^{-12}$ \\
0.25 & -0.01020310975429537 & 0.16997595759445022 & $3.399442879969960 \times 10^{-11}$ \\
0.5 & -0.007496876529468979 & 0.19646732842074352 & $6.365820370834996 \times 10^{-11}$ \\
0.75 & -0.004099110842952412 & 0.22314095416570680 & $8.448242105885129 \times 10^{-12}$ \\
1 & $5.9121871734293 \times 10^{-8}$ & 0.25000002937629756 & $2.485910088889653 \times 10^{-10}$ \\
\hline
\end{tabular}

Table 4. Approximate solution $\bar{y}(t ; r)$ of fourth order VIM at $t=0.5$ and $r \in[0,1]$

\begin{tabular}{cccc}
\hline $\mathrm{r}$ & $\underline{c_{2}}(r)$ & $\underline{y}(0.5 ; r)$ & {$[\underline{E}(0.5)]_{r}$} \\
\hline 0 & 0.023538969063634813 & 0.35935808190205626 & $7.0343751656931630 \times 10^{-9}$ \\
0.25 & 0.016554919244886100 & 0.33172332776916430 & $3.9969035997566850 \times 10^{-9}$ \\
0.5 & 0.010307281584441760 & 0.30428777924960454 & $2.0336278494514910 \times 10^{-9}$ \\
0.75 & 0.004810786651353893 & 0.27704784239391295 & $8.636787535154511 \times 10^{-10}$ \\
1 & $5.9121871734293 \times 10^{-8}$ & 0.25000002937629756 & $2.485910088889653 \times 10^{-10}$ \\
\hline
\end{tabular}

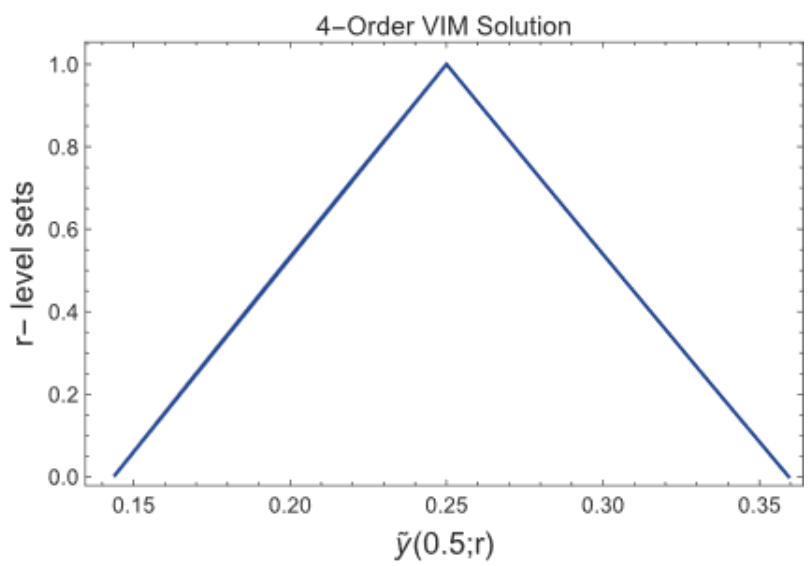

Figure 4. VIM approximate solution of (13) at $t=0.5$ and $r \in[0,1]$

According to Tables 3-4 and Figure 4, we concluded that the fourth-order VIM approximate solutions of (7) for all $t \in[0,1]$ and $r \in[0,1]$ comply with the fuzzy numbers of properties as triangular fuzzy number.

Example 5.2 [35]: Consider this non-homogenous second-order non-linear TPFBVP:

$$
\begin{aligned}
& \tilde{y}^{\prime \prime}(t)+\tilde{y}(t)=\tilde{y}^{3}(t)+\tilde{f}(t), t \in[0,1] \\
& \tilde{y}(0 ; r)=\tilde{a}, \tilde{y}(1)=\tilde{b}
\end{aligned}
$$

where $\tilde{a}=\left[\frac{1}{4}\left(\frac{r-2}{10}\right)+\frac{1}{4}, \frac{1}{4}\left(\frac{2-r}{10}\right)+\frac{1}{4}\right]$ and $\tilde{b}=\left[\frac{5}{4}\left(\frac{r-2}{10}\right)+\frac{e^{-1}}{4}, \frac{5}{4}\left(\frac{2-r}{10}\right)+\frac{e^{-1}}{4}\right]$ for all $r \in[0,1]$. According to [30] the fuzzy function $\tilde{f}(t)$ have the following defuzzification:

$$
\tilde{f}(t ; r)=\left[\left(t+\frac{1}{4}\right)\left(\frac{r-2}{10}\right)-\frac{e^{-t}}{4}\right]^{3}+\left(t+\frac{1}{4}\right)\left(\frac{2-r}{10}\right),\left[\left(t+\frac{1}{4}\right)\left(\frac{2-r}{10}\right)-\frac{e^{-t}}{4}\right]^{3}+\left(t+\frac{1}{4}\right)\left(\frac{r-2}{10}\right),
$$

Then the corresponding analytic solution of (13) is given by

$$
\tilde{Y}(t ; r)=\frac{e^{-t}}{4}+\left(t+\frac{1}{4}\right)\left(\frac{r-2}{10}, \frac{r-2}{10}\right)
$$

by following the initial guesses from Example 5.1, we have:

$$
\left\{\begin{array}{l}
\underline{y}_{0}(t ; r)=\frac{1}{4}\left(\frac{r-2}{10}\right)+\frac{1}{4}+\underline{c}_{2}(r) t, \\
\bar{y}_{0}(t ; r)=\frac{1}{4}\left(\frac{2-r}{10}\right)+\frac{1}{4}+\bar{c}_{2}(r) t .
\end{array}\right.
$$




\subsection{HPM formulation}

From Section 4 the approximate solution of (13) can be determine by HPM from the followings:

$$
\begin{aligned}
& p^{0}:\left\{\underline{y}_{0}(t ; r)=\frac{1}{4}\left(\frac{r-2}{10}\right)+\frac{1}{4}+\underline{c}_{2}(r) t,\right. \\
& p^{1}:\left\{\begin{array}{c}
\underline{y}_{1}(t ; r)=\underline{L}_{2}^{-1}\left[\underline{y}_{0}(t ; r) 1+\left(\underline{y}_{0}\left(t ; r ; \underline{c}_{2}(r)\right)\right)^{3}+\underline{f}(t ; r)\right], \\
\underline{y}_{1}(0 ; r)=0, \underline{y}_{1}(1 ; r)=0 .
\end{array}\right. \\
& \vdots \\
& p^{k+1}:\left\{\begin{array}{r}
y_{k+1}(t ; r)=\underline{L}_{2}^{-1} \underline{y}_{k}(t ; r)+\sum_{i=0}^{k-1} \sum_{j=0}^{i} \underline{y}_{k-1-i}\left(t ; r ; \underline{c}_{2}(r)\right) \underline{y_{j}}\left(t ; r ; \underline{c}_{2}(r)\right) \underline{y_{i-j}}\left(t ; r ; \underline{c}_{2}(r)\right), \\
\underline{y}_{k}(0 ; r)=0, \underline{y}_{k}(1 ; r)=0 .
\end{array}\right.
\end{aligned}
$$

Similarly for the upper bound

$$
\begin{aligned}
& p^{0}:\left\{\bar{y}_{0}(t ; r)=\frac{1}{4}\left(\frac{2-r}{10}\right)+\frac{1}{4}+\bar{c}_{2}(r) t,\right. \\
& p^{1}:\left\{\begin{array}{c}
\bar{y}_{1}(t ; r)=\bar{L}_{2}^{-1}\left[\bar{y}_{0}(t ; r)+\left(\bar{y}_{0}\left(t ; r ; \bar{c}_{2}(r)\right)\right)^{3}+\bar{f}(t ; r)\right], \\
\bar{y}_{1}(0 ; r)=0, \bar{y}_{1}(1 ; r)=0 .
\end{array}\right. \\
& p^{k+1}:\left\{\begin{array}{c}
\bar{y}_{k+1}(t ; r)=\underline{L}_{2}^{-1} \bar{y}_{k}(t ; r)+\sum_{i=0}^{k-1} \sum_{j=0}^{i} \bar{y}_{k-1-i}\left(t ; r ; \underline{c}_{2}(r)\right) \bar{y}_{j}\left(t ; r ; \underline{c}_{2}(r)\right) \bar{y}_{i-j}\left(t ; r ; \underline{c}_{2}(r)\right) \\
\bar{y}_{k}(0 ; r)=0, \bar{y}_{k}(1 ; r)=0 .
\end{array}\right.
\end{aligned}
$$

Next, the determination of the values of $\tilde{c}_{2}(r)$ for all $r \in[0,1]$ can be done by solving the nonlinear seventh order HPM series solution of (17) subject to the fuzzy boundary condition $\underline{y}(1 ; r)=\frac{5}{4}\left(\frac{r-2}{10}\right)+\frac{e^{-1}}{4}$ and $\bar{y}(1 ; r)=\frac{5}{4}\left(\frac{2-r}{10}\right)+\frac{e^{-1}}{4}$ of $(13)$. Then, we substitute the values of $\tilde{c}_{2}(r)$ again in the seventh order series solution of (13) to obtain seventh order HPM series solution in Table 5.

Table 5. Approximate solution of seventh order HPM of (13) at $t=0.5 \forall r \in[0,1]$

\begin{tabular}{ccccc}
\hline $\mathrm{r}$ & $\underline{c}_{2}(r)$ & $\bar{c}_{2}(r)$ & $\underline{y}(0.5 ; r)$ & $\bar{y}(0.5 ; r)$ \\
\hline 0 & -0.44985575506896760 & -0.04891373031802654 & 0.0016323384080985234 & 0.30163258314132690 \\
0.25 & -0.42489220652617230 & -0.07406371025083800 & 0.0203823093927938160 & 0.28288260102272034 \\
0.5 & -0.39991573435157324 & -0.09920243697696327 & 0.0391322807152672800 & 0.26413260400295260 \\
0.75 & -0.37492630650497016 & -0.12432965360921329 & 0.0578822454883946360 & 0.24538262188434600 \\
1 & -0.34992391523689814 & -0.14944512128297516 & 0.0766322059390083700 & 0.22663263231515884 \\
\hline
\end{tabular}

\subsection{VIM formulation}

The VIM to solve (13) is in compliance with section 4. The formulation shall be given as in (18):

$$
\left\{\begin{array}{c}
\underline{y}_{i+1}\left(t ; r ; \underline{c}_{2}(r)\right)=\underline{y}_{i}\left(t ; r ; \underline{c}_{2}(r)\right) \\
+\int_{0}^{t}[\eta-t]\left\{\begin{array}{c}
\underline{y}_{i}^{\prime \prime}\left(\eta ; r ; \underline{c}_{2}(r)\right)-\underline{y}_{i}\left(t ; r ; \underline{c}_{2}(r)\right) \\
-\underline{y}_{i}\left(\eta ; r ; \underline{c}_{2}(r)\right)^{3}-\underline{f}(t ; r)
\end{array}\right\} d \eta, \\
\bar{y}_{i+1}\left(t ; r ; \bar{c}_{2}(r)\right)=\bar{y}_{i}\left(t ; r ; \bar{c}_{2}(r)\right) \\
+\int_{0}^{t}[\eta-t]\left\{\begin{array}{c}
\bar{y}_{i}^{\prime \prime}\left(\eta ; r ; \bar{c}_{2}(r)\right)-\bar{y}_{i}\left(t ; r ; \bar{c}_{2}(r)\right) \\
-\bar{y}_{i}\left(\eta ; r ; \bar{c}_{2}(r)\right)^{3}-\bar{f}(t ; r)
\end{array}\right\} d \eta .
\end{array}\right.
$$

By following VIM formulation and analysis in Example 5.2, third-order VIM series solution is given in the following Table 6: 
Table 6. Approximate solution of third order VIM of (13) at $t=0.5 \forall r \in[0,1]$

\begin{tabular}{ccccc}
\hline $\mathrm{r}$ & $\underline{c_{2}}(r)$ & $\bar{c}_{2}(r)$ & $\underline{y}(0.5 ; r)$ & $\bar{y}(0.5 ; r)$ \\
\hline 0 & -0.4500000805284215 & -0.05000128774459855 & 0.076632688439284070 & 0.30163199259313456 \\
0.25 & -0.4250000216571309 & -0.07500108858114996 & 0.057882686548291370 & 0.28288209737819590 \\
0.5 & -0.3999999813340253 & -0.10000090368612918 & 0.039132675010097500 & 0.26413219449035540 \\
0.75 & -0.3749999595833066 & -0.12500073372107165 & 0.020382653821232450 & 0.24538228361308428 \\
1 & -0.3499999563806096 & -0.15000057928658794 & 0.001632623001234793 & 0.22663236446104182 \\
\hline
\end{tabular}

In order to show efficiency of VIM and HPM methods in solving (13), numerical comparisons of the accuracy generated by RKHS in [35] are presented in Table 7. These comparisons are conducted at $t=0.5$ and various $r$ fuzzy level that belongs to $[0,1]$ by computing the mean of the average error. Here, $\overline{\tilde{E}(t ; r)}$ is the mean of the average error between $\underline{E}(t ; r)$ and $\bar{E}(t ; r)$ such that

$$
\left\{\begin{array}{l}
\underline{E}(t ; r)=|\underline{Y}(t ; r)-\underline{y}(t ; r)|, \\
\bar{E}(t ; r)=|\bar{Y}(t ; r)-\bar{y}(t ; r)| .
\end{array}\right.
$$

Table 7. Numerical comparison of mean errors $\overline{\widetilde{E}(t ; r)}$ at $t=0.5$

\begin{tabular}{cccc}
\hline $\mathrm{r}$ & Third-order VIM & Seventh order HPM & RKHS [33] \\
\hline 0 & $1.267224325474103 \times 10^{-7}$ & $2.397203478878614 \times 10^{-7}$ & $3.87851 \times 10^{-6}$ \\
0.25 & $1.690374040186348 \times 10^{-7}$ & $2.369393627074407 \times 10^{-7}$ & $3.31263 \times 10^{-7}$ \\
0.5 & $2.251369623368360 \times 10^{-7}$ & $2.521985155955197 \times 10^{-7}$ & $1.84790 \times 10^{-7}$ \\
0.75 & $2.948819071355024 \times 10^{-7}$ & $2.508378323807635 \times 10^{-8}$ & $1.34477 \times 10^{-7}$ \\
1 & $3.780944354508757 \times 10^{-8}$ & $2.630438642283513 \times 10^{-8}$ & $1.09977 \times 10^{-7}$ \\
\hline
\end{tabular}

According to Tables 6 and 7 and Figure 5, we concluded that the third order VIM and seventh order HPM will successfully provide the approximate solutions to (7) for all $t \in[0,1]$ and $r \in[0,1]$. This is shown by the results that comply with the fuzzy numbers of properties as triangular fuzzy number.

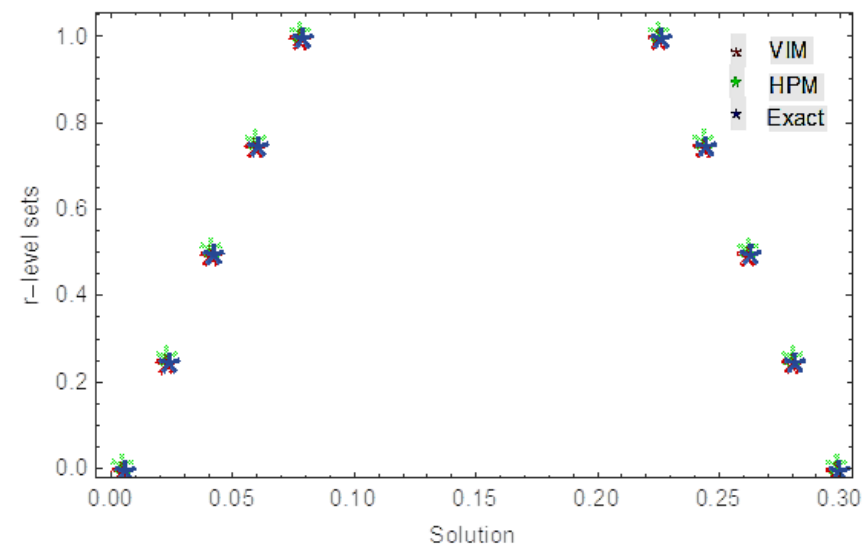

Figure 5. Exact solution of (13) compared with third order VIM and seventh order HPM approximate solution of (13) at $t=0.5$ and $r \in[0,1]$

\section{RESULTS COMPARISON}

In this section, we present a comparative explanation between the solutions of nonlinear TPFBVPs obtained by HPM and VIM as we illustrate from Examples 5.1 and 5.2:

- The initial approximation guesses in HPM, and VIM are obtained in the same way.

- The construction of VIM formula to solve nonlinear TPFBVP is faster and easier than HPM because HPM takes the advantage of the small parameter $p \in[0,1]$ that makes HPM suffers from the cumbersome work needed for the derivation of for nonlinear terms. This will increase the computational work especially when the degree of nonlinearity increases. 
- As mentioned in section 4, VIM is used directly without any requirement or restrictive assumptions that the nonlinear terms make the series solution longer and time consuming in CPU. The third order VIM solved (7) within 31.2264607 seconds for reach $r$-level values. In HPM the use of embedding parameter $p$ is decomposed for the nonlinear terms making the series solution shorter than the solution of VIM with less time consuming in CPU. The seventh order HPM solved (7) within 1.4882989 seconds for all $r$-level values. For illustration, the nonlinear term $y^{2}$ in (7) which has been decomposed to $\sum_{k=0}^{n-1} y_{k} y_{n-1-k}$ in HPM formulation but in VIM formula we substitute $y^{2}$.

- From the results obtained by HPM in Tables 1-2 and VIM in Tables 3-4, we conclude that VIM provides a better and more accurate solution than HPM, with less order of series solution for both $t \in[0,1]$ and $r \in[0,1]$

- Finally, both Figure 3 and Figure 4 show the solution of (7) by using HPM and VIM respectively for all $t \in[0,1]$ and $r \in[0,1]$ satisfy the fuzzy numbers properties in the form of triangular fuzzy number.

\section{CONCLUSION}

In this work, approximate analytical methods have been used for nonlinear TPFBVPs to achieve an approximate solution. Two schemes, HPM and VIM, were developed and reformulated to approximate the nonlinear TPFBVP solution. Numerical examples, including nonlinear TPFBVPs, demonstrate the efficacy of these approaches. Forgiven nonlinear TPFBVPs, a comparison of HPM and VIM results was presented. The comparison shows that VIM convergence is faster and provides an improved solution, particularly for the less approximate terms, nonlinear TPFBVPs over HPM. Even though these equations are without exact analytical solutions, the exactness of both HPM and VIM can be calculated from nonlinear TPFBVPs. The VIM Lagrangian multiplier for the nonlinear TPFBVPs is equivalent to the value for all the r-level sets. All outcomes of the experiments with HPM and VIM are achieved using a triangular shape to acquire the properties of the fuzzy numbers.

\section{ACKNOWLEDGEMENT} Sains Malaysia.

All authors gratefully acknowledge the facilities and financial assistance provided by Universiti

\section{REFERENCES}

[1] S. Tomasiello, S. K. Khattri, and J. Awrejcewicz, "Differential quadrature-based simulation of a class of fuzzy damped fractional dynamical systems," International Journal of Numerical Analysis and Modeling, vol. 14, no. 1, pp. 63-75, 2017.

[2] M. Mazandarani and L. Xiu, "A review on fuzzy differential equations," IEEE Access, vol. 9, pp. 62195-62211, 2021, doi: 10.1109/ACCESS.2021.3074245.

[3] D. E. Sánchez, V. F. Wasques, J. P. Arenas, E. Esmi, and L. C. de Barros, "On interactive fuzzy solutions for mechanical vibration problems," Applied Mathematical Modelling, vol. 96, pp. 304-314, Aug. 2021, doi: 10.1016/j.apm.2021.03.002.

[4] B. Bede, "A note on 'two-point boundary value problems associated with non-linear fuzzy differential equations'," Fuzzy Sets and Systems, vol. 157, no. 7, pp. 986-989, Apr. 2006, doi: 10.1016/j.fss.2005.09.006.

[5] F. S. Pedro, L. C. de Barros, and E. Esmi, "Population growth model via interactive fuzzy differential equation," Information Sciences, vol. 481, pp. 160-173, May 2019, doi: 10.1016/j.ins.2018.12.076.

[6] Ö. Akin and Ö. Oruç, "A prey predator model with fuzzy initial values," Hacettepe Journal of Mathematics and Statistics, vol. 41, no. 3, pp. 387-395, 2012.

[7] S. Tapaswini and S. Chakraverty, "Numerical solution of fuzzy arbitrary order predator-prey equations," Applications and Applied Mathematics: An International Journal, vol. 8, no. 2, pp. 647-672, 2013.

[8] L. C. de Barros, R. C. Bassanezi, and W. A. Lodwick, Studies in Fuzziness and Soft Computing A First Course in Fuzzy Logic, Fuzzy Dynamical Systems, and Biomathematics Theory and Applications, vol. 347. Berlin, Heidelberg: Springer Berlin Heidelberg, 2017

[9] S. S. Devi and K. Ganesan, "Modelling electric circuit problem with fuzzy differential equations," Journal of Physics: Conference Series, vol. 1377, Nov. 2019, Art. no. 012024, doi: 10.1088/1742-6596/1377/1/012024.

[10] M. S. El Naschie, "From experimental quantum optics to quantum gravity via a fuzzy Kähler manifold," Chaos, Solitons and Fractals, vol. 25, no. 5, pp. 969-977, Sep. 2005, doi: 10.1016/j.chaos.2005.02.028.

[11] N. Bildik and A. Konuralp, "The use of variational iteration method, differential transform method and adomian decomposition method for solving different types of nonlinear partial differential equations," International Journal of Nonlinear Sciences and Numerical Simulation, vol. 7, no. 1, Jan. 2006, doi: 10.1515/IJNSNS.2006.7.1.65.

[12] T. Allahviranloo and K. Khalilpour, "A numerical method for two-point fuzzy boundary value problems," World Applied Sciences Journal, vol. 13, no. 10, pp. 2137-2147, 2011

[13] K. Khalilpour and T. Allahviranloo, "An initial-value method for two-point fuzzy boundary value problems," World Applied Sciences Journal, vol. 13, no. 10, pp. 2148-2155, 2011

[14] X. Guo, D. Shang, and X. Lu, "Fuzzy approximate solutions of second-order fuzzy linear boundary value problems," Boundary Value Problems, vol. 2013, no. 1, p. 212, Dec. 2013, doi: 10.1186/1687-2770-2013-212.

[15] A. F. Jameel, N. R. Anakira, A. K. Alomari, D. M. Alsharo, and A. Saaban, "New semi-analytical method for solving two point nth order fuzzy boundary value problem," International Journal of Mathematical Modelling and Numerical Optimisation, vol. 9 , no. 1, pp. 12-31, 2019, doi: 10.1504/IJMMNO.2019.096906. 
[16] N. R. Anakira, A. Jameel, A. K. Alomari, A. Saaban, M. Almahameed, and I. Hashim, "Approximate solutions of multipantograph type delay differential equations using multistage optimal homotopy asymptotic method," Journal of Mathematical and Fundamental Sciences, vol. 50, no. 3, pp. 221-232, Dec. 2018, doi: 10.5614/j.math.fund.sci.2018.50.3.1.

[17] J. H. He, "Application of homotopy perturbation method to nonlinear wave equations," Chaos, Solitons and Fractals, vol. 26, no. 3, pp. 695-700, Nov. 2005, doi: 10.1016/j.chaos.2005.03.006.

[18] J.-H. He, "Homotopy perturbation method for bifurcation of nonlinear problems," International Journal of Nonlinear Sciences and Numerical Simulation, vol. 6, no. 2, Jan. 2005, doi: 10.1515/IJNSNS.2005.6.2.207.

[19] J.-H. He, "Approximate solution of nonlinear differential equations with convolution product nonlinearities," Computer Methods in Applied Mechanics and Engineering, vol. 167, no. 1-2, pp. 69-73, Dec. 1998, doi: 10.1016/S0045-7825(98)00109-1.

[20] L. Xu, "Variational principles for coupled nonlinear Schrödinger equations," Physics Letters A, vol. 359, no. 6, pp. 627-629, Dec. 2006, doi: 10.1016/j.physleta.2006.07.026.

[21] Y. Molliq R, M. S. M. Noorani, and I. Hashim, "Variational iteration method for fractional heat- and wave-like equations," Nonlinear Analysis: Real World Applications, vol. 10, no. 3, pp. 1854-1869, Jun. 2009, doi: 10.1016/j.nonrwa.2008.02.026.

[22] S. Narayanamoorthy and S. Mathankumar, "Variational iterative method: an appropriate numerical scheme for solving system of linear Volterra fuzzy integro-differential equations," Advances in Difference Equations, vol. 2018, no. 1, p. 394, Dec. 2018, doi: 10.1186/s13662-018-1829-y.

[23] M. A. Noor, K. I. Noor, and S. T. Mohyud-Din, "Variational iteration method for solving sixth-order boundary value problems," Communications in Nonlinear Science and Numerical Simulation, vol. 14, no. 6, pp. 2571-2580, Jun. 2009, doi: 10.1016/j.cnsns.2008.10.013.

[24] J. H. He, "Approximate analytical solution for seepage flow with fractional derivatives in porous media," Computer Methods in Applied Mechanics and Engineering, vol. 167, no. 1-2, pp. 57-68, Dec. 1998, doi: 10.1016/S0045-7825(98)00108-X.

[25] D. Dubois and H. Prade, "Towards fuzzy differential calculus part 3: Differentiation," Fuzzy Sets and Systems, vol. 8, no. 3, pp. 225-233, Sep. 1982, doi: 10.1016/S0165-0114(82)80001-8.

[26] Z. Akbarzadeh Ghanaie and M. Mohseni Moghadam, "Solving fuzzy differential equations by runge-kutta method," Journal of Mathematics and Computer Science, vol. 02, no. 02, pp. 208-221, Feb. 2011, doi: 10.22436/jmcs.002.02.01.

[27] S. Seikkala, "On the fuzzy initial value problem," Fuzzy Sets and Systems, vol. 24, no. 3, pp. 319-330, Dec. 1987, doi: 10.1016/0165-0114(87)90030-3

[28] O. S. Fard, "An iterative scheme for the solution of generalized system of linear fuzzy differential equations," World Applied Sciences Journal, vol. 7, no. 12, pp. 1597-1604, 2009.

[29] L. A. Zadeh, "Toward a generalized theory of uncertainty (GTU)- An outline," Information Sciences, vol. 172, no. 1-2, pp. 1-40, Jun. 2005, doi: 10.1016/j.ins.2005.01.017.

[30] B. Bede, "Note on 'Numerical solutions of fuzzy differential equations by predictor-corrector method," Information Sciences, vol. 178, no. 7, pp. 1917-1922, Apr. 2008, doi: 10.1016/j.ins.2007.11.016.

[31] F. Rabiei, F. Ismail, A. Ahmadian, and S. Salahshour, "Numerical solution of second-order fuzzy differential equation using improved runge-kutta nystrom method," Mathematical Problems in Engineering, vol. 2013, pp. 1-10, 2013, doi: $10.1155 / 2013 / 803462$.

[32] D. D. Ganji and A. Sadighi, "Application of homotopy-perturbation and variational iteration methods to nonlinear heat transfer and porous media equations," Journal of Computational and Applied Mathematics, vol. 207, no. 1, pp. 24-34, Oct. 2007, doi: 10.1016/j.cam.2006.07.030.

[33] J. H. He, "Homotopy perturbation technique," Computer Methods in Applied Mechanics and Engineering, vol. 178, no. 3-4, pp. 257-262, Aug. 1999, doi: 10.1016/S0045-7825(99)00018-3.

[34] T. Allahviranloo, S. Abbasbandy, and S. S. Behzadi, "Solving nonlinear fuzzy differential equations by using fuzzy variational iteration method," Soft Computing, vol. 18, no. 11, pp. 2191-2200, Nov. 2014, doi: 10.1007/s00500-013-1193-5.

[35] O. A. Arqub, M. Al-Smadi, S. Momani, and T. Hayat, "Application of reproducing kernel algorithm for solving second-order, two-point fuzzy boundary value problems," Soft Computing, vol. 21, no. 23, pp. 7191-7206, Dec. 2017, doi: 10.1007/s00500-0162262-3. 